\title{
Prostate-specific antigen as a diagnostic tool: how useful and reliable is it?
}

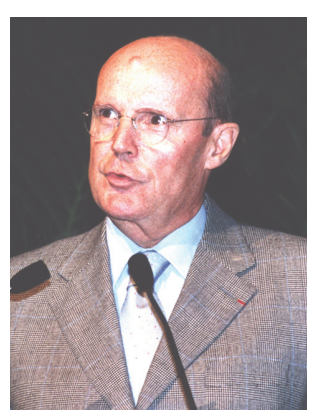

Laurent Boccon-Gibod CHU Bichat, Department of Urology, CHU Bichat, APHP, 75018, Paris, France laurent.boccongibod@bch.ap-hop-paris.fr future, part of fso

\author{
'While definitely not a tumor \\ marker, PSA has been used and \\ approved as a marker for the \\ detection of prostate cancer...'
}

Prostate-specific antigen (PSA), a kallikrein isolated by Wang in 1979 [1], is a physiologic excretory product of the healthy prostatic epithelium, involved in the semen liquefaction process. A small amount of PSA (less than 1 molecule per million excreted) leaks into the bloodstream where its normal level was - arbritarily - fixed more than 2 decades ago at $4 \mathrm{ng} / \mathrm{ml}$ and shown to rise in any prostatic pathologic condition associated with alterations of the basement membrane: prostatitis, benign prostatic hypertrophy (BPH) and, of course, cancer. While definitely not a tumor marker, PSA has been used and approved as a marker for the detection of prostate cancer [1] and therefore, extensively promoted as the marker for early detection and eventually, screening. This has induced an unprecedented revolution in urology and oncology, leading to a spectacular increase in the incidence of prostate cancer, inducing a dramatic expansion of surgery and radiation therapy without any spectacular modification of the prostate cancer death rate up to this point in time. However, once initially seen as a 'magic bullet', PSA is increasingly confronting clinicians with more questions than they seem able to answer, which this editorial will try to summarize.

Is there a 'normal' PSA level below which patients can be assured that they do not have prostate cancer?

The answer given by the Prostate Cancer Prevention Trial (PCPT [2]), where all patients underwent a biopsy at the end of the trial, is negative, as $8 \%$ of the patients with PSA less than $1 \mathrm{ng} / \mathrm{ml}$ harbored cancer, $8 \%$ of which were poorly differentiated. This explains the continuous and increasing tendency to lower PSA thresholds triggering prostate biopsies, eventually down to $2.5 \mathrm{ng} / \mathrm{ml}$ for instance; however, the consequences of such an attitude are not negligible as this would lead to the biopsy of 1.8 million American men, with an $80 \%$ negative biopsy rate, associated with a spectacular increase in the detection of indolent/potentially unsignificant tumors, the treatment of which induces significant side effects [3].

\section{Is there a level of PSA at which the diagnosis of prostate cancer is highly probable?}

The answer a definitive yes when the PSA is greater than $15 / 20 \mathrm{ng} / \mathrm{ml}$, however, the majority of the patients present with a PSA at 15 or below 15 , and the presence of a relatively large volume of BPH is more often that not the cause of the PSA elevation [4], so that PSA has even been proposed as a surrogate for digital rectal examination of the evaluation of prostate volume.

\section{If PSA in itself is not reliable, can more} sophisticated assays be of clinical use? PSA occurs in the bloodstream in various forms [1]: free PSA, mostly produced by benign tissue, has now been divided into benign PSA (bPSA), intact PSA (iPSA) and pro-PSA. PSA can be complexed to alpha-chymo-thrypsin, macroglobulin and $\alpha-1$ protease inhibitor.

$\therefore$...PSA has even been proposed as a surrogate for digital rectal examination in the evalution of prostate volume.'

The most widely used of these forms, also known as 'PSA derivatives' is undoubtedly free PSA as well as the ratio of free to total PSA expressed as percent-free PSA. In routine clinical practice, it should be remembered that the percent-free PSA is only really clinically relevant at extreme values; below $10 \%$, it is highly in favor of the presence of prostate cancer, and above $30 \%$, it is more predictive of benign disease. But inflammation can significantly reduce the percent-free PSA and more importantly, the great majority of patients seen in the clinic, have a percent-free PSA in the gray zone somewhere between 15 and $25 \%$, therefore, although it has been shown to be a good 
discriminant between benign and malignant prostate conditions, not only in the range of 4-10 total PSA but also in the range $2-4$, it is difficult, except as previously mentioned, in the extreme values, to use percent-free PSA to make an informed decision as to whether or not to perform a prostate biopsy at the individual level. Complex PSA has not been proven to be truly superior to free PSA among the various forms of free PSA, and BPSA is apparently related essentially to $\mathrm{BPH}$, whereas Pro-PSA would be more cancer specific.

As the assay for pro-PSA will be available this year, the results are eagerly awaited.

\section{'...the interpretation of PSA kinetics should be based on a baseline PSA value taken before the age of 40 , because later on the development of $\mathrm{BPH}$ induces a very significant confounding factor...}

The ratio of PSA to overall prostate volume (PSA density) or to the volume of the transition (PSA TZ density), measured by ultrasound, have shown to be of some value in certain studies; however, it should be pointed out that ultrasound prostate measurements are often operator dependant and may not always be $100 \%$ reliable.

If a single value of PSA is not informative enough and considering that early localized prostate cancer is a slow growing tumor, should one rely on serial PSA measurements over time to assess PSA kinetics, rather than on a single PSA determination?

Setting aside the methodological issues related to the measurements of PSA velocity and doubling time [5], it has been shown that to be accurate, the interpretation of PSA kinetics should be based on a baseline PSA value taken before the age of 40 , because later on the development of BPH induces a very significant confounding factor and, indeed, a PSA velocity of $0.2 \mathrm{ng} / \mathrm{ml}$, which has been considered to be predictive of death by prostate cancer [6] and has also been demonstrated to be a determinant of future $\mathrm{BPH}$ progression. The use of PSA kinetics in the monitoring of patients enrolled in a program of surveillance with delayed treatment is to a lesser degree - the diagnosis of cancer having been made - associated with the same difficulties [7].
Because PSA is not a tumor marker, it cannot in itself be hoped to be the magic bullet for the early detection/screening of prostate cancer

PSA will surely in the not too distant future be replaced by cancer-specific markers [8,9], however, none of those currently available in the research literature are really ready for 'prime time'. PSA therefore remains one of the elements to be included in a diagnostic strategy, which should include age, family history, voiding symptoms, clinical examination (digital rectal examination) and, eventually, sophisticated imaging studies such as magnetic resonance imaging of the prostate. The wide and discriminate use of PSA testing should definitely be discouraged, particularly before the age of 40 and above the age of 75 [10].

\section{Once the diagnosis of prostate cancer has been made, there is indeed a relationship between the level of PSA and probably percent free PSA and tumor volume, as well as the grade of the tumor and thus to the prognosis}

However, if this is true for high levels of PSA (above $15 \mathrm{ng} / \mathrm{ml}$ ), it does not constantly prove true for levels under $10 \mathrm{ng} / \mathrm{ml}$, and total PSA as well as free PSA cannot by themselves be used as isolated prognostic markers and here again, should be integrated in a multimodal strategy of treatment decisions [1].

\section{Once the cancer has been treated, PSA becomes an extremely solid and reliable marker of treatment efficacy}

After treatment with curative intent, be it surgery or radiation therapy, PSA relapse indeed implies treatment failure, but is not in itself predictive of the final outcome, which is closely related to PSA kinetics [11-13].

After palliative treatment with androgen suppression for metastatic disease, the nadir PSA is highly predictive of response duration, and patients with a nadir below $0.2 \mathrm{ng} / \mathrm{ml} \mathrm{usu-}$ ally enjoy a very significant progression-free survival [14].

In conclusion, neither PSA itself nor its derivatives are a diagnostic tumor marker for the early diagnosis and screening of prostate cancer.

The use of PSA should be intregrated in a multimodal diagnostic strategy in order to avoid a nonacceptable increase of negative biopsies associated with a potentially significant morbidity and worrisome overtreatment of potentially unsignificant/indolent prostate cancers. 
On the contrary, once the cancer has been treated, PSA remains at this point in time, the best marker of treatment efficacy.

\section{Financial \& competing interests disclosure}

The authors have no relevant affiliations or financial

involvement with any organization or entity with a financial interest in or financial conflict with the subject matter or materials discussed in the manuscript. This includes employment, consultancies, honoraria, stock ownership or options, expert testimony, grants or patents received or pending, or royalties.

No writing assistance was utilized in the production of this manuscript.

\section{Bibliography}

1. Stephan C, Jung K, Lein M, Diamandis EP: PSA and other kallikreins for prostate cancer detection. Eur. J. Cancer 43, 1918-1926 (2007).

2. Thompson IM, Pauler DK, Goodman PJ et al: : Prevalence of prostate cancer among men with a PSA level equal of inferior to 4.0 ng/ml. N. Engl. J. Med. 350, 2239-2246 (2004).

3. Welch HG, Schwartz LM, Woloshin S: Prostate-specific antigen levels in the United States: implications of various definitions for abnormal. J. Natl Cancer Inst. 97, 1132-1137 (2005).

4. Stamey TA, Caldwell M, Mc Neal JE, Nolley R, Ermonez M, Downs J: The prostate specific antigen era in the United States is over for prostate cancer: what happened in the last 20 years? J. Urol. 172, 1297-1301 (2004).

5. Daskivich TJ, Regan MM, Oh WK: Prostate specific antigen doubling time calculation: not as easy as 1, 2, 4. J. Urol. 176, 1927-1937 (2006).
6. Loeb S, Roehl KA, Catalona WJ, Nadler RB: PSA velocity threshold for predicting prostate cancer in young men. J. Urol. 177 , 899-902 (2007).

7. Klotz LH: Active surveillance with selective delayed intervention in localized prostate cancer. Can. J. Urol. 12 (supl1) 53-55 (2005).

8. Fradet Y, Saad F, Aprikian A et al:: UPM3 a new molecular urine test for the detection of prostate cancer. Urology 64, 311-316 (2004).

9. Leman ES, Cannon GW, Trock BJ et al: EPCA-2: a highly specific serum marker for prostate cancer. Urology. 69, 714-720 (2007).

10. Kerfoot BP, Holmberg EF, Lawler EV, Krupat E, Conlin PR: Practioner-level determinants of inappropriate prostatespecific antigen screening. Arch Intern Med. 167, 1367-1372 (2007).

11. Stephenson AJ, Kattan MW, Eastham JA et al:: Defining biochemical recurrence of prostate cancer after radical prostatectomy: a proposal for a standardized definition. J. Clin. Oncol. 24, 3973-3978 (2006).
12. Roach M 3rd, Hanks G, Thames H Jr et al:: Defining biochemical failure following radiotherapy with or without hormonal therapy in men with clinically localized prostate cancer: recommendations of the RTOG-ASTRO Phenix Consensus Conference. Int J Radiat Oncol Biol Phys. 65, 965-974 (2006).

13. Lee AK, D'Amico AV: Using prostatespecific antigen doubling time in clinical practice. BJU Int. 100, 243-244 (2007).

14. Hussain M, Tangen CM, Higano C et al: Absolute prostate-specific antigen value after androgen deprivation is a strong independent predictor of survival in new metastatic prostate cancer: data from Southwest Oncology Group Trial 9346 (INT-0162). J. Clin. Oncol. 24, 3984-3990 (2006).

\section{Affiliation}

- Laurent Boccon-Gibod CHU Bichat, Department of Urology, $C H U$ Bichat, APHP, 75018, Paris, France laurent.boccon-gibod@bch.ap-hop-paris.fr 